Current Science International

Volume: 10 | Issue: 04| Oct. - Dec.| 2021

\author{
EISSN:2706-7920 ISSN: 2077-4435 \\ DOI: $10.36632 / \mathrm{csi} / 2021.10 .4 .59$ \\ Journal homepage: www.curresweb.com \\ Pages: 705-714
}

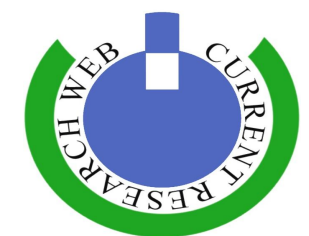

\title{
Feeding Practices, Nutritional and Socioeconomic Status Impact on Caries Experience among Group of Sudanese Children
}

\author{
Rabab M. Abd Elhakam ${ }^{1}$, Mohammed Abou el Yazid ${ }^{2}$, Nayera E. Hassan ${ }^{3}$, Nancy A. \\ Fakhry $^{4}$ and Tamer M. Abd Elwahab ${ }^{5}$
}

${ }^{1}$ Assistant Researcher, Orthodontics and Pediatric Dentistry Department, National Research Centre, 33 El Buhouth St., 12622 Dokki, Giza, Egypt.

${ }^{2}$ Prof. of Pediatric Dentistry, Head of Orthodontics and Pediatric Dentistry Department, National Research Centre, 33 El Buhouth St., 12622 Dokki, Giza, Egypt.

${ }^{3}$ Prof. of Biological Anthropology, Head of Biological Anthropology Department, National Research Centre, 33 El Buhouth St., 12622 Dokki, Giza, Egypt.

${ }^{4}$ Prof. of Physical Anthropology, Anthropology Department, Faculty of African Postgraduate Studies, Cairo University, Egypt.

${ }^{5}$ Lecturer of Physical Anthropology, Anthropology Department, Faculty of African Postgraduate Studies, Cairo University, Egypt.
Received: 24 June 2021
Accepted: 15 July 2021
Published: 30 Nov. 2021

\begin{abstract}
Background: According to the WHO, "breastfeeding is the normal way of providing young infants with the nutrients they need for healthy growth and development. Exclusive breastfeeding is recommended up to 6 months of age, with continued breastfeeding along with appropriate complementary foods up to two years of age or beyond". However, several studies have reported prolonged and unrestricted breastfeeding as a potential risk factor for primary tooth caries. Understanding the role that breast feeding and bottle-feeding play in the development of dental caries during childhood is essential in helping dentists and parents and care providers prevent the disease, and also for the development of effective public health policies. However, the issue is not yet fully understood. Aim: to evaluate the impact of feeding practices, nutritional status and socioeconomic status on caries experience. Methods: A cross-sectional study was conducted in the Sudanese nurseries and schools in Cairo, Egypt. A total 320 child of both sex were examined, they aged from 1 to 4 years. Feeding practice data were collected from them through a face-to-face interview. Dental examination was done using the dmft index according to the WHO criteria. Nutritional assessment was assessed by recording patient's anthropometric measures (weight and height). Socioeconomic status assessment was done using Fahmy modified index that was designed for evaluation of the social status of families, to be used in health research in Egypt. Results: Breastfed children had experienced caries more than bottle fed ones. $91.8 \%$ of the children who experienced caries were fed on need (irregular feeding). A moderate positive significant correlation was found between $\mathrm{dmft}$ and age $(\mathrm{r}=0.41)$; the older the child the more caries occurrence. A weak negative significant correlation was found out between $\mathrm{dmft} \&$ nutritional status $(\mathrm{r}=-0.28)$, a weak positive significant correlation between $\mathrm{dmft} \&$ socioeconomic status $(\mathrm{r}=0.31)$ as most of the children who experienced caries were high and medium SES. Conclusion: Children who experienced caries were breastfed and were weaned at age of 2 years. The higher the socioeconomic status of a child, the more caries experience. Increased frequency of feeding practice may increase the risk of dental caries.
\end{abstract}

Keywords: feeding practice, breast feeding, bottle feeding, caries, nutritional status, socioeconomic status, nocturnal feeding.

Corresponding Author: Rabab M. Abd Elhakam, Orthodontics and Pediatric Dentistry Department, National Research Centre, Dokki, Giza, Egypt. E-mail: dr.rabab_nrc@hotmail.com 


\section{Introduction}

According to the WHO, "breastfeeding is the normal way of providing young infants with the nutrients they need for healthy growth and development. Exclusive breastfeeding is recommended up to 6 months of age, with continued breastfeeding along with appropriate complementary foods up to two years of age or beyond". However, several studies have reported prolonged and unrestricted breastfeeding as a potential risk factor for primary tooth caries (Paglia, 2015).

Understanding the role that breast feeding and bottle-feeding play in the development of dental caries during childhood is essential in helping dentists and parents and care providers prevent the disease, and also for the development of effective public health policies. However, the issue is not yet fully understood (Avila et al., 2015).

Dental caries is an infectious, transmissible, bacterial disease affecting children and adults of all races, ethnicities and socio-economic levels. It is a major public health problem both within the U.S. and around the world, and has devastating effects including pain, infection, nutritional insufficiencies, learning and speech problems, and even death (Kierce et al., 2016)

An individual's socioeconomic status (SES) is one of the most important determinants in children's oral health. Previous studies found out that children with low SES, including low household income, low mother's education and living in socially disadvantaged families, were more likely to have higher prevalence of dental caries and greater dental pain experience (Zhang et al., 2021).

Nutritional assessment can be defined as the interpretation from dietary, laboratory, anthropometric, and clinical studies. It is used to determine the nutritional status of individual or population groups as influenced by the intake and utilization of nutrients (Gibson, 2005).

Nutritional assessment is the quantitative evaluation of nutritional status. A comprehensive nutritional assessment has five components:

-Dietary, medical and medication history

-Physical examination

-Growth, anthropometric and body composition measurements (Phillips and Jensen, 2019).

Dietary choices affect oral health as well as general health and well-being. Establishment of a dental home by 12 months of age allows the institution of individualized caries-preventive strategies, including dietary recommendations and appropriate oral hygiene instruction, as the primary teeth begin to erupt (AAPD, 2017).

So, Aim of this study is to investigate the impact of feeding practices, nutritional and socioeconomic status on caries experience among a sample of Egyptian children aged 1-4 years.

\section{Subjects and methods}

A cross-sectional study was carried out. A total 320 Sudanese children (168 boys and 152 girls) were examined. Children aged 1 to 4 years and were recruited from four Sudanese schools and nurseries in Cairo Governorate. Accurate birthday was available from either the parent supported by birth certificates or from the school registrant (personal school chart for each child). Children were classified to 3 groups, group1 (from 12 to 23 months), group 2 (from $>23$ to $<35$ months) and group 3 (from 35 to $\leq 48$ months).

\section{Ethical consideration}

The study was approved by The Ethical Committee of the National Research Centre (registration no. 18110).

The guardians (either the father or the mother) were asked to give written informed consent to participate in the study after explanation of the study aim and examination procedures. The data pertaining to the subjects were entered into the database with a numerical code only.

\section{Anthropometric measures (Nutritional status assessment)}

Weight and length were assessed using standardized equipment and follows the recommendation of international biological program (WHO, 2013). 


\section{- Measurement of weight}

The Weight was measured to the nearest $0.1 \mathrm{~kg}$ by using a digital balance machine. The child weighted with minimum clothes, no shoes and without touching anything (Krishna et al., 2017).

\section{- Measurement of length}

Length was measured to children $<2$ years, while height is measured to children $>2$ years. The length was measured by infantometer, while the height was measured to the nearest $0.1 \mathrm{~cm}$ using the ordinary measuring tape fixed at the wall and the child standing up after removing the shoes with feet parallel to each other and pointed forward and the back is straight in upright position. The knees must be straight and the head in position that Frankfort Plane must be horizontal (Krishna et al., 2017).

Weight, height(length), age and, sex data were used to calculate Z-scores of the nutritional indicators (WHZ ) in comparison to the published World Health Organization/National Center for Health Statistics (WHO/ NCHS) reference population using the WHO Anthro-Plus Software (Version 1.0.4) (WHO, 2009).

The nutritional status was classified according to the World Health Organization Classification of weight for height $Z$ score (2007) as follows:

-Very thin: $\mathrm{WHZ}<-3 \mathrm{SD}$.

-Underweight: $\mathrm{WHZ}<-2$ SD. Percentile: $0<3$.

$\cdot$ Normal: $W H Z \geq-2$ and $\leq+1$ SD. Percentile: $\geq 3$ and $\leq 85$.

-Overweight: $\mathrm{WHZ}>+1$ and $\leq+2$ SD. Percentile $>85$ and $\leq 97$.

-Obese: $\mathrm{WHZ}>+2$ SD. Percentile $>97$

\section{Socioeconomic status assessment:}

The Socioeconomic status of the participants was determined using Fahmy modified index (Fahmy et al., 2015). This index was designed for evaluation of the social status of families, to be used in health research in Egypt.

Data were collected by the examiner, using an interview questionnaire that included the following updated social Questions:
1. Mother's education.
6. Per-capita income.
2. Father's education.
7. Family size.
3. Working status of the mother.
8. Crowding index.
4. Working status of the father.
5. Use of computer
9. Proper sewage disposal.
10. Proper refuse disposal.

The examiner asked the questions to one /or both of the parents of each child and filled the index as well. School children, whom parents were not met, were telephoned interviewed.

The total score of the scale was 54, and according to the final score, the family was classified into: high, medium and low SES as the following:

High $\geq 75 \%$ ( 40.5 points). Medium 50 to $<75 \%$ ( $<40.5>27$ points). Low $<50 \%$ ( $<27$ points).

\section{Dental examination}

Dental examination of the children was carried out by the dentist using torch light, disposable mirror, and explorer. Dental caries was measured using the decayed (d), missing (m), and filled (f) teeth $(\mathrm{dmft})$ index according to the WHO criteria. Teeth missing $(\mathrm{m})$ or filled $(\mathrm{f})$ contributed to the overall $\mathrm{dmft}$ score only if teeth were missing or filled because of dental caries. After dmft calculation; two dmft groups were obtained; with caries group $(\mathrm{dmft}=\mathrm{or}>1)$ and caries free group $(\mathrm{dmft}=0)$.

- Young children (1 to 2 years) were examined using knee-to-knee examination.

- Older children (2 to 4 years) were seated on a small chair during the dental examination.

\section{Statistical analysis}

All Data were collected, tabulated and subjected to statistical analysis. Statistical analysis was performed with SPSS 20®1, Graph Pad Prism ${ }^{\circledR 1}$, and Microsoft Excel 20163. All quantitative data presented as mean \& standard deviation, while qualitative data were presented as frequency \& percentages, all data presented in tables \& figures. Comparisons two different groups were performed 
by using Independent t-test. All comparisons in qualitative data were performed by using Chi square test. Spearman's correlation coefficient was performed to evaluate all correlations. Results is significant when $\mathrm{p}$-value $<0.05$.

\section{Results}

The study sample consisted of 320 children aged from 1 to 4 years (168 boys and 152 girls). For each child: dmft was recorded, weight and length were measured, and nutritional status and SES were assessed.

Frequency and percentages of dmft (caries free and with caries) among different age group are shown in table (1). Comparison between caries and no caries in different age groups revealed significant difference as $\mathrm{P}<0.05$ (caries free higher than with caries), as presented in table (1). Also, correlation was calculated by using Spearmen's correlation coefficient which revealed moderate positive significant correlation between $\mathrm{dmft} \&$ age $(\mathrm{r}=0.41)$.

Table 1: Comparison and correlation between frequency \& percentages of dmft categories among different age groups:

\begin{tabular}{|c|c|c|c|c|c|c|c|}
\hline \multirow[b]{3}{*}{ Age Group (months) } & \multirow[b]{3}{*}{ Total } & \multicolumn{6}{|c|}{ dmft } \\
\hline & & \multicolumn{2}{|c|}{ No Caries } & \multicolumn{2}{|c|}{ With Caries } & \multirow{2}{*}{$P$ value } & \multirow{2}{*}{$\mathbf{r}$} \\
\hline & & $\mathbf{N}$ & $\%$ & $\mathbf{N}$ & $\%$ & & \\
\hline 12 to $>24$ & 73 & 73 & 100.0 & 0 & 0.0 & $0.0001 *$ & \multirow{4}{*}{0.41 * } \\
\hline 24 to $>36$ & 100 & 95 & 95.0 & 5 & 5.0 & $0.0001 *$ & \\
\hline 36 to $>48$ & 147 & 89 & 60.5 & 58 & 39.5 & $0.006^{*}$ & \\
\hline Total & 320 & 257 & 80.3 & 63 & 19.7 & $0.0001 *$ & \\
\hline
\end{tabular}

Frequency \& percentages of dmft (caries free and with caries) among sex is shown in table (2). Comparison between dmft (caries free and with caries) among sex revealed insignificant difference in (with caries) as $\mathrm{P}>0.05$, while revealed significant difference in (caries free) as $\mathrm{P}<0.05$, as presented in table (2).

Table 2: Comparison between Frequency \& percentages of dmft categories distribution among sex:

\begin{tabular}{cccccccc}
\hline & & \multicolumn{5}{c}{ Gender } & \multirow{2}{*}{ P Value } \\
\cline { 2 - 6 } dmft & Total & Boys & $\mathbf{N}$ & $\mathbf{\%}$ & $\mathbf{N}$ & $\mathbf{\%}$ & \\
\hline Caries free & 257 & 142 & 55.3 & 115 & 44.7 & $0.01^{*}$ \\
With Caries & 63 & 26 & 41.3 & 37 & 58.7 & 0.06 \\
\hline
\end{tabular}

Frequency \& percentages of dmft (caries free and with caries) among different nutritional statuses (thin and underweight, normal, overweight and obese) is shown in table (3). Comparison between 4 categories of nutritional status in both $\mathrm{dmft}$ categories was performed by using Chi square test which revealed significant difference between them as $\mathrm{P}<0.05$ in both categories of dmft. Normal was significantly the highest in both $\mathrm{dmft}$ categories while (Thin and underweight) was significantly the lowest in (no caries) \& (Obese) in (with caries), as presented in table (3). Also, correlation was calculated by using Spearmen's correlation coefficient which revealed weak negative significant correlation between $\mathrm{dmft} \&$ nutritional status $(\mathrm{r}=-0.28)$.

Table 3: Comparison and correlation between Frequency \& percentages of dmft categories among different nutritional statuses:

\begin{tabular}{|c|c|c|c|c|c|c|c|c|c|c|c|}
\hline \multirow[b]{3}{*}{ dmft } & \multirow[b]{3}{*}{ Total } & \multicolumn{8}{|c|}{ Nutritional status } & \multirow{3}{*}{$\underset{\text { Value }}{P}$} & \multirow{3}{*}{$\mathbf{r}$} \\
\hline & & \multicolumn{2}{|c|}{$\begin{array}{c}\text { Thin and } \\
\text { underweight }\end{array}$} & \multicolumn{2}{|c|}{ Normal } & \multicolumn{2}{|c|}{ Overweight } & \multicolumn{2}{|c|}{ Obese } & & \\
\hline & & $\mathbf{N}$ & $\%$ & $\mathbf{N}$ & $\%$ & $\mathbf{N}$ & $\%$ & $\mathbf{N}$ & $\%$ & & \\
\hline Caries free & 257 & 11 & 4.2 & 194 & 75.6 & 26 & 10.1 & 26 & 10.1 & $0.001^{*}$ & - \\
\hline With Caries & 63 & 21 & 33.3 & 37 & 58.7 & 0 & 0.0 & 5 & 8.0 & $0.001 *$ & $0.28^{*}$ \\
\hline
\end{tabular}

Frequency \& percentages of dmft (caries free and with caries) among different socioeconomic status (low, medium and high) is shown in table (4). Comparison between 3 categories of 
socioeconomic standard in both $\mathrm{dmft}$ categories was performed by using Chi square test which revealed significant difference between them as $\mathrm{P}<0.05$. (Medium) was significantly the highest while (Low) was significantly the lowest in both dmft categories, as presented in table (4). Also, correlation was calculated by using Spearmen's correlation coefficient which revealed weak positive significant correlation between $\mathrm{dmft} \&$ socioeconomic status $(\mathrm{r}=0.31)$.

Table 4: Comparison and correlation between Frequency \& percentages of dmft categories among different socioeconomic status:

\begin{tabular}{|c|c|c|c|c|c|c|c|c|c|}
\hline \multirow[b]{3}{*}{ dmft } & \multirow[b]{3}{*}{ Total } & \multicolumn{6}{|c|}{ Socioeconomic status } & \multirow{3}{*}{ P Value } & \multirow{3}{*}{$\mathbf{r}$} \\
\hline & & \multicolumn{2}{|c|}{ Low } & \multicolumn{2}{|c|}{ Medium } & \multicolumn{2}{|c|}{ High } & & \\
\hline & & $\mathbf{N}$ & $\%$ & $\mathbf{N}$ & $\%$ & $\mathbf{N}$ & $\%$ & & \\
\hline Caries free & 560 & 53 & 20.6 & 131 & 51.0 & 73 & 28.4 & $0.001^{*}$ & ก $21 *$ \\
\hline With Caries & 110 & 5 & 7.9 & 16 & 25.4 & 42 & 66.7 & $0.001 *$ & $0.31^{*}$ \\
\hline
\end{tabular}

\section{Comparison between frequency and percentages of $\mathrm{dmft}$ categories among different answers regarding feeding practice questions}

Frequency and percentages of dmft (caries free and with caries) among different types of feeding practices (breast feeding, bottle feeding and combination) is shown in table (5). There was a significant difference between all answers in both categories of $\mathrm{dmft}$, in both categories (breast feeding) was significantly the highest while (bottle feeding) was significantly the lowest in (no caries) category while in (with caries) category there was insignificant difference between (bottle feeding) \& (combination).

Table 5: Comparison between frequency and percentages of different answers regarding the question what was the type of feeding practice?

\begin{tabular}{|c|c|c|c|c|c|c|c|c|}
\hline \multirow{3}{*}{$\begin{array}{l}\text { Sudanese } \\
\text { dmft }\end{array}$} & \multirow[b]{3}{*}{ Total } & \multicolumn{6}{|c|}{ Question 1 what was the type of feeding practice? } & \multirow{3}{*}{ P Value } \\
\hline & & \multicolumn{2}{|c|}{ Breast feeding } & \multicolumn{2}{|c|}{ Bottle feeding } & \multicolumn{2}{|c|}{ Combination } & \\
\hline & & $\mathbf{N}$ & $\%$ & $\mathbf{N}$ & $\%$ & $\mathbf{N}$ & $\%$ & \\
\hline Caries free & 257 & 141 & 54.8 & 58 & 22.6 & 58 & 22.6 & $0.001^{*}$ \\
\hline With Caries & 63 & 53 & 84.1 & 0 & 0 & 10 & 15.9 & $0.001 *$ \\
\hline
\end{tabular}

Frequency \& percentages of dmft (caries free and with caries) in different weaning timing (one year, two year and still feeding) is shown in table (6). Comparison between different answers revealed significant difference between them as $\mathrm{P}<0.05$ in (with caries) while there was insignificant difference between them in (no caries). (Two years) was significantly the highest while there was insignificant difference between (one year) \& (still feeding) in both dmft categories, as presented in table (6).

Table 6: Comparison between Frequency \& percentages of different answers regarding the question when was the time of weaning?

\begin{tabular}{|c|c|c|c|c|c|c|c|c|}
\hline \multirow{3}{*}{ dmft } & \multirow{3}{*}{ Total } & \multicolumn{7}{|c|}{ Question: when was the time of weaning? } \\
\hline & & \multicolumn{2}{|c|}{ One year } & \multicolumn{2}{|c|}{ Two years } & \multicolumn{2}{|c|}{ Still feeding } & \multirow{2}{*}{ P value } \\
\hline & & $\mathbf{N}$ & $\%$ & $\mathbf{N}$ & $\%$ & $\mathbf{N}$ & $\%$ & \\
\hline Caries free & 257 & 79 & 30.7 & 99 & 38.6 & 79 & 30.7 & 0.06 \\
\hline With Caries & 63 & 0 & 0.0 & 63 & 100.0 & 0 & 0.0 & $0.0001^{*}$ \\
\hline
\end{tabular}

Frequency and percentages of dmft (caries free and with caries) and frequency of feeding (every 2-3 hours and on need) is shown in table (7). Comparison between different answers revealed significant difference by using Chi square test in both categories of dmft as $\mathrm{P}<0.05$, (on need) answer was significantly higher than (Every 2-3 hours) in both categories, as presented in table (7).

Frequency and percentages of dmft (caries free and with caries) among different types of feeding at bedtime (breast feeding, bottle feeding and combination) is shown in table (8). Comparison between different answers revealed significant difference by using Chi square test in both categories of $\mathrm{dmft}$ as $\mathrm{P}<0.05$, (breast feed) was significantly the highest, while (combination) was significantly the lowest in both categories, as presented in table (8). 
Table 7: Comparison between Frequency \& percentages of distribution of dmft regarding the question how many times do you feed your child?

\begin{tabular}{|c|c|c|c|c|c|c|}
\hline \multirow{3}{*}{ dmft } & \multirow{3}{*}{ Total } & \multicolumn{5}{|c|}{ Question: how many times do u feed your child? } \\
\hline & & \multicolumn{2}{|c|}{ Every 2 to 3 hours } & \multicolumn{2}{|c|}{ On need } & \multirow{2}{*}{$P$ value } \\
\hline & & $\mathbf{N}$ & $\%$ & $\mathbf{N}$ & $\%$ & \\
\hline Caries free & 257 & 21 & 8.2 & 236 & 91.8 & $0.0001^{*}$ \\
\hline With Caries & 63 & 21 & 33.3 & 42 & 66.7 & $0.002 *$ \\
\hline
\end{tabular}

Table 8: Comparison between Frequency \& percentages of distribution of dmft regarding the question what was the type of feeding at bedtime?

\begin{tabular}{|c|c|c|c|c|c|c|c|c|}
\hline \multirow{3}{*}{ dmft } & \multirow{3}{*}{ Total } & \multicolumn{6}{|c|}{ Question: at bedtime what was the type of feeding? } & \multirow{3}{*}{$P$ value } \\
\hline & & \multicolumn{2}{|c|}{ Breast feed } & \multicolumn{2}{|c|}{ Bottle feed } & \multicolumn{2}{|c|}{ Combination } & \\
\hline & & $\mathbf{N}$ & $\%$ & $\mathbf{N}$ & $\%$ & $\mathbf{N}$ & $\%$ & \\
\hline Caries free & 257 & 147 & 57.1 & 68 & 26.6 & 42 & 16.3 & $0.001 *$ \\
\hline With Caries & 63 & 58 & 92.0 & 5 & 8.0 & 0 & 0.0 & $0.0001 *$ \\
\hline & \multicolumn{3}{|c|}{ Grading Standards } & \multicolumn{3}{|c|}{ Correlation Degree } & & \\
\hline & & \multicolumn{2}{|c|}{$\begin{array}{c}\rho=0 \\
0<|\rho| \leq 0.19 \\
0.20 \leq|\rho| \leq 0.39 \\
0.40 \leq|\rho| \leq 0.59 \\
0.60 \leq|\rho| \leq 0.79 \\
0.80 \leq|\rho| \leq 1.00 \\
1.00\end{array}$} & \multicolumn{2}{|c|}{$\begin{array}{c}\text { no correlation } \\
\text { very week } \\
\text { weak } \\
\text { moderate } \\
\text { strong } \\
\text { very strong } \\
\text { monotonic correlation }\end{array}$} & & & \\
\hline
\end{tabular}

\section{Discussion}

Feeding practices during infancy play a major role in causation of ECC because teeth are more vulnerable for caries immediately after eruption. Night feeding during sleep provides fermentable carbohydrates. This, along with diminished salivary flow at night, increases the risk of ECC. There is evidence in the medical literature to conclude night feeding with formula milk, adding sugar to formula milk and excessive consumption of sweets will promote ECC (Perera et al., 2014).

Feeding habits, particularly during the first year of life, involve numbers of simultaneous and interrelated behaviors, such as breastfeeding, bottle-feeding and the introduction of complementary foods (Feldens et al., 2018). The World Health Organization (WHO) recommended that mothers should breastfeed babies exclusively for the first 6 months and then continue breastfeeding along with other weaning foods for up to 2 years or later (WHO, 2021).

This study was designed to evaluate the impact of feeding practice, nutritional status and socioeconomic status on caries experience. Also to assess sex differences in caries experience. A total 320 child aged from 1 to 4 years (168 boys and 152 girls) was examined.

Caries free children were $80.3 \%$ of the total examined children; $54.8 \%$ were breast fed. Our findings is consisted with some studies that we reviewed showed no effect of Breast feeding on dental caries (Nunes et al., 2012 and Kramer et al., 2009).

Children who had experienced caries were $19.7 \%$ of the sample; $84.1 \%$ were breastfed and $15.9 \%$ were on combination. Breastfed children had experienced caries more than bottle fed ones that goes with Perera et al. (2014) who also revealed an increased risk of ECC among children exclusively breast fed up to six months, but the increase was not statistically significant. In contrast to our findings, the results of two other systematic reviews showed that any breastfeeding, in general, is protective against dental caries compared to children who never received breast milk (Avila et al., 2015; Cui et al., 2017). In contrast to Al Dashti et al. (1995) in Kuwait who found that children on combination (breast and bottle) were less caries experienced than bottlefed.

It was found out that caries experience is significantly positive correlated with age $(\mathrm{r}=0.41)$; the older the child the more caries occurrence; this finding is consisted with Chanpum et al. (2020) in Thailand, Elamin et al., (2018) in United Arab Emirates and Ferreira et al., (2007).

Our findings showed that there is significant difference between boys and girls in caries free group where $80.3 \%$ of the children were caries free. $55.3 \%$ were boys and $44.7 \%$ were girls. Boys 
were significantly higher than girls in caries free group; and that was in contrast with Abu Hamila, 2013 who found out that ECC significantly affects males more than females.

While $19.7 \%$ of the sample was experiencing caries; $41.3 \%$ were boys while $58.7 \%$ were girls with no significant diffrences and that were consisted with Ferreira et al. (2007). Also a study was done by Bafti et al. (2015) in Iran who found out that dmft was higher in boys compared to girls.

Exploring the relation between nutritional status and dental caries is controversial (Edalat et al., 2014). Undeniably, it is likely that not a single common risk factor plays a role in the relationship between ECC and BMI but rather a complex interface of dietary pattern, oral hygiene practices, and genetic aspects that determine both caries and alterations in BMI. We found out a weak negative significant correlation between $\mathrm{dmft} \&$ nutritional status $(\mathrm{r}=-0.28)$; and that was similar to studies done by Krishna et al. (2017) in India and Ambarkova and Bakracevska, (2015) in Republic of Macedonia.

$75.6 \%$ of the caries free children were normal nutritional status. While $58.7 \%$ of the children who experienced caries were normal nutritional, $33.3 \%$ were thin and underweight. In contrast to Ahmed and Abuaffan, (2015) in Sudan who found out that higher prevalence of dental caries in primary teeth were found in underweight groups. Also Koksal et al., 2011 in turkey who found out those Children with low body weight have a higher risk of developing dental caries than overweightobese children. He also found out that maximum number of children were underweight, which might be due to the social class difference among children and study setting done in schools (Public and private).

We found out a weak positive significant correlation between $\mathrm{dmft} \&$ socioeconomic status $(\mathrm{r}=0.31) ; 51 \%$ of the caries free children were medium SES, $28.4 \%$ high SES, and $20.6 \%$ low SES. While $66.7 \%$ of the children who experienced caries were high SES, $25.4 \%$ were medium and $7.9 \%$ were low SES. Most of the children who experienced caries were high SES and that was similar to a study conducted by Prasanth et al. (2011) where a greater number of caries was found in private school group people who are from upper socioeconomic status. It could be because soft drinks and chocolates, which are promptly open, and oftentimes devoured, speak to high sugar source that may add to the potential for dental caries.

In contrast to other studies that showed that children from lower SES families suffer from more severe dental pain, higher prevalence of dental caries and dental fluorosis (Zhou et al., 2018 and Peres et al., 2019). A study was done by Sukumaran et al. (2020) who found out that there was no correlation between dental caries, BMI, and SES among Private preschool children.

In contrast to our findings, Devenish et al. (2020) who did not observe an association between prolonged full or any breastfeeding for $>12$ months and increased caries risk. Also Victora et al., 2016 who stated that breastfeeding in itself and of prolonged duration has been shown to be beneficial for many health outcomes, such as overweight, diabetes, and infections. We found out $38.6 \%$ of the caries free children were weaned at age of 2years, 30.7\%were weaned at 1 year and $30.7 \%$ were still feeding. While children who experienced caries; all were weaned at age of 2 years. Two studies found a significant association between being breastfed for $>12$ or 24 months and dental caries (Feldens et al., 2010; Chaffee et al., 2014. Also Hong et al. (2014) Tanaka et al. (2013) had all shown an increase in dental caries with increased duration of breast feeding.

A study was done by Nirunsittirat et al. (2016) who observed a dose-response relationship between the frequency of sleeping while breast- or bottle-feeding and dmfs. Also higher frequency of any infant feeding may increase the risk of dental caries as stated by Feldens et al. (2018). We found out that $91.8 \%$ of the children who experienced caries were fed on need while $8.2 \%$ were fed regularly (every 2 to 3 hours). $66.7 \%$ of the children who experienced caries were fed on need and $33.3 \%$ were fed every 2 to 3 hours. increased the frequency, which points towards the theory that increased feeding frequency might be the major explanation for the observed association between prolonged breastfeeding and dental caries; which was proved by van Meijeren-van Lunteren et al. (2021) that the proportion of children with dental caries was clearly higher among infants who had been exposed to increased breastfeeding frequency at the age of 12 months. Also a study was done by Feldens et al. (2010) who stated that Daily breastfeeding frequency was associated with S-ECC in a previous study of Brazilian preschoolers, in which breastfeeding frequency, but not duration $\geq 12$ months, maintained statistical significance in multi-variable models. 
A study was done by Nakayama and Mori, (2015) who found out significant associations between ECC and nocturnal breastfeeding. We found out $57.1 \%$ of the caries free children were breastfed at bedtime, $26.6 \%$ were bottle-fed at bedtime and $16.3 \%$ were on combination at bedtime. $19.7 \%$ of the Sudanese patients were suffering from caries; $92 \%$ were breastfed at bedtime and $8 \%$ were bottle-fed at bedtime. Breastfed children at night experienced more caries than those bottle-fed ; that is may be clarified by the theory that during the night, the salivary flow is less, leading to decreased re-mineralization which increases the risk of developing caries lesions over time (Shelton et al., 1977; Reisine and Psoter, 2001). In contrast to our findings, the results of 2 other systematic reviews showed that any breastfeeding, in general, is protective against dental caries compared to children who never received breast milk (Avila et al., 2015; Cui et al., 2017). Also van Meijeren-van Lunteren et al. (2021) who found that nocturnal bottle-feeding is related to an increased caries risk.

\section{Limitations of the study:}

The challenge we faced was data collection during the pandemic Covid-19 virus.

\section{Conflict of interests}

There was no conflict of interests in this study.

\section{Conclusion}

1- Children who experienced caries; Breastfed ones experienced caries more than other types feeding practices.

2- Increased frequency of feeding practice increases the risk of dental caries.

3- Children who experienced caries; were weaned at age of 2 years.

4- The older the child the more caries occurrence.

5- There was no sex differences in caries experience in caries group.

6- There was a positive correlation between SES and caries experience, the higher the socioeconomic status of a child, the more caries experience.

7- There was negative correlation between caries experience and nutritional status.

\section{Recommendations:}

1- Mothers with low levels of education may require special attention because their children are of greater risk of caries and would benefit most from preventive efforts.

2- The extent of oral health education and regular training of the mothers to perform cleaning of the teeth for their children by dentists and health care givers should be encouraged.

\section{References}

Abu Hamila, N.A., 2013. Early childhood caries and certain risk factors in a sample of children 1-3.5 years in Tanta. Dentistry, 4:180 10.4172/2161-1122.1000180

Ahmed, T.E., and A.H. Abuaffan, 2015. Correlation between body mass index and dental caries among a sample of Sudanese children. Braz. Dent. Sci., 18:42-1.

American Academy on Pediatric Dentistry Council on Clinical Affairs (AAPD), 2017. Policy on dietary recommendations for infants, children, and adolescents. Pediatr. Dent., 30:47-8.

Al-Dashti A.A., S.A. Williams and M.E. Curzon, 1995. Breast feeding, bottle feeding and dental caries in Kuwait, a country with low-fluoride levels in the water supply. Community Dent Health, 12(1):42-7.

Ambarkova, V., and G. Bakracevska, 2015. Relating Dental Caries Experience with Body Mass Index among Primary School Children from Ohrid City - A Pilot Study. Aperito. J. Oral. Health Dent., $1: 1-3$.

Avila W.M., I.A. Pordeus, S.M. Paiva and C.C. Martins, 2015. Breast and Bottle Feeding as Risk Factors for Dental Caries: A Systematic Review and Meta-Analysis PLoS One, 18; 10(11): $\mathrm{e} 0142922$.

Bafti, L.S., M.A. Hashemipour, H. Poureslami, and Z. Hoseinian, 2015. Relationship between Body Mass Index and Tooth Decay in a Population of 3-6-Year-Old Children in Iran. Int. J. Dent., 2015:126530. 
Chaffee, B.W., C.A. Feldens, and M.R. Vitlo, 2014. Association of long-duration breastfeeding and dental caries estimated with marginal structuralmodels. Ann. Epidemiol., 24(6):448-54.

Chanpum, P., D. Duangthip, C. Trairatvorakul, and S. Songsiripradubboon, 2020. Early Childhood Caries and Its Associated Factors among 9- to 18-Month Old Exclusively Breastfed Children in Thailand: A Cross-Sectional Study. Int. J. Environ. Res. Public Health,May 4; 17(9):3194.

Cui, L., X. Li, Y. Tian, J. Bao, L. Wang, and D. Xu, 2017. Breastfeeding and early childhood caries: a meta- analysis of observational studies. Asia. Pac. J. Clin Nutr., 26(5): 867-80.

Devenish, G., A. Mukhtar, A. Begley, A.J. Spencer, and W.M. Thomson, 2020. Early childhood feeding practices and dental caries among Australian preschoolers The American Journal of Clinical Nutrition, 111(44): 821-828.

Edalat, A., M. Abbaszadeh, M. Eesvandi, and A. Heidari, 2014. The Relationship of Severe Early Childhood Caries and Body Mass Index in a Group of 3- to 6-year-old Children in Shiraz. J Dent (Shiraz). Jun, 15(2):68-73.

Elamin A, M Garemo, and A. Gardner, 2018. Dental caries and their association with socioeconomic characteristics, oral hygiene practices and eating habits among preschool children in Abu Dhabi, United Arab Emirates - the NOPLAS project. BMC Oral Health. Jun 8; 18(1):104.

Fahmy, S., L. Nofal, S. Shehata, H. El Kady, and H. Ibrahim, 2015. Updating indicators for scaling the socioeconomic level of families for health research. Journal of the Egyptian Public Health Association, 90: 1-7.

Ferreira, S.H., J.U. Beria, P.F. Kramer, E.G. Feldens, and C.A. Feldens, 2007. Dental Caries in 0-5 year-old Brazilian Children: Prevalence, Severity and associated factors. Int. J. of Pediatr. Dent., 17: 289-296.

Feldens, C.A., E.R.J. Giugliani, A. Vigo, and M.R. Vitolo, 2010. Early feeding practices and severe early childhood caries in four-year-old children from southern Brazil: a birth cohort study. Caries Res., 44: 445-52.

Feldens, C.A., P.H. Rodrigues, G. de Anastácio, M.R. Vítolo, and B.W. Chaffee, 2018. Feeding frequency in infancy and dental caries in childhood: a prospective cohort study. Int. Dent. J. Apr; 68(2):113-121.

Gibson R.S., 2005. Principles of Nutritional Assessment Oxford University Press. 2005. https://books.google.com.eg/books/about/Principles_of_Nutritional_Assessment.html?id=lBlu7U $\mathrm{KI} 3 \mathrm{aQC} \&$ redir_esc $=\mathrm{y}$

Hong, C.H., R.A. Bagramian, S.M. HashimNainar, L.H. Straffon, L. Shen, and C.Y. Hsu, 2014. High caries prevalence and risk factors among young preschool children in an urban community with water fluoridation. Int. J. Paediatr. Dent., 24:32-42.

Kierce, E.A., L.D. Boyd, L. Rainchuso, C.A. Palmer, and A. Rothman, 2016. Association between Early Childhood Caries, Feeding Practices and an Established Dental Home J. Dent. Hyg., 90 (1):18-27.

Koksal, E., M. Tekcicek, S.S. Yalcin, B. Tugrul, S. Yalcin, and G. Pekcan, 2011. Association between anthropometric measurements and dental caries in Turkish school children. Cent. Eur. J. Public Health, 19:147-51.

Kramer, M.S., L. Matush, and N. Bogdanovich, 2009. Health and development outcomes in 6.5-y-old children breastfed exclusively for 3 or 6 mo. Am. J. Clin. Nutr., 90:1070-4.

Krishna, HVNS, E. Manaswini, V.Y. Kumar, P. Bellamkonda, ASK Bhargava, R.R. Jaidupally, 2017. Association between Nutritional Status and Early Childhood Caries in Indian Children. J Int Soc Prev Community Dent. 2017 May-Jun; 7(3):131-135.

Nakayama, Y., and M. Mori, 2015. Association between nocturnal breastfeeding and snacking habits and the risk of early childhood caries in 18- to 23-month-old Japanese children. J. Epidemiol., 25(2):142-7.

Nunes, A.M., C.M. Alves, and F. Borba de Araujo, 2012. Association between prolonged breastfeeding and early childhood caries: a hierarchical approach. Community Dent. Oral Epidemiol., 40:542-9.

Paglia, L., 2015. Does breastfeeding increase risk of early childhood caries? Eur. J. Paediatr. Dent., 16(3):173. 
Perera, P.J., M.P. Fernando, T.D. Warnakulasooriya and N. Ranathunga, 2014. Effect of feeding practices on dental caries among preschool children: hospital based analytical cross sectional study. Asia. Pac. J. Clin. Nutr., 23(2):272-7.

Peres, K.G., G.G. Nascimento, M.A. Peres, M.N. Mittinty, F.F. Demarco, I.S. Santos, A. Matijasevich and A.J.D. Barros, 2017. Impact of Prolonged Breastfeeding on Dental Caries: A PopulationBased Birth Cohort Study Pediatrics, 140(1).

Philips, S.M. and C. Jensen, 2021 Indications for nutritional assessment in childhood. https:/ /www. uptodate.com

Prasanth, S.T., B. Venkatesh, and V.D. Kumar, 2011. Comparison of dental caries in relation with body mass index (BMI) in government and private school children. J. Dent. Sci. Res., 2(2):1-5.

Reisine S.T. and W. Psoter, 2001. Socioeconomic status and selected behavioral determinants as risk factors for dental caries. J. Dent. Educ., 65(10):1009-16.

Shelton, P.G., R.J. Berkowitz, and D.J. Forrester, 1977. Nursing bottle caries. Pediatrics. May; 59(5): $777-8$.

Tanaka, K., Y. Miyake, S. Sasaki, and Y. Hirota, 2013. Infant feeding practices and risk of dental caries in Japan: the Osaka Maternal And Child Health Study. Pediatr., Dent., 35(3):267-71.

Van Meijeren-van Lunteren, A.W., T. Voortman, M.E.C. Elfrink, E.B. Wolvius, and L. Kragt, 2021. Breastfeeding and Childhood Dental Caries: Results from a Socially Diverse Birth Cohort Study. Caries Res., 55(2):153-161.

Victora, C.G., R. Bahl, A.J. Barros, G.V. Franca, S. Horton, and J. Krasevec, 2016. Breastfeeding in the 21st century: epidemiology,mechanisms and lifelong effect. Lancet., 387(10017): 475-90.

WHO, 2009. WHO Anthroplus for personal computers manual: software for assessing growth of the world's children and adolescents'. Geneva: World Health Organization Press; http://www.who.int/childgrowth/software/anthro_pc_manual.pdf.

WHO, 2013. Expert Committee on Biological Standardization Sixtieth report. WHO Technical Report Series, No. 977, (viii and 231 pages) Web site: www.who.int/biologicals

WHO, Infant and young child feeding https://www.who.int/news-room/fact-sheets/detail/infant-andyoung-child-feeding.

Zhang, T., J. Hong, X. Yu, Q. Liu, A. Li, Z. Wu, and X. Zeng, 2021. Association between socioeconomic status and dental caries among Chinese preschool children: a cross-sectional national study. BMJ Open. May 21; 11(5):e042908.

Zhou, Y., D.R. Chen, and Q.H. Zhi, 2018. The prevalence and associated risk indicators of dental fluorosis in China: findings from the 4th national oral health survey. Chin. J. Dent. Res., 21:20511. 